\title{
Novel measurement techniques with annular arrays for scanning acoustic microscopy
}

\author{
Sebastian Kümmritz, André Juhrig, Lukas Timmermann, Elfgard Kühnicke \\ Technische Universität Dresden, Mommsenstraße 15, 01069 Dresden, Germany, \\ sebastian.kuemmritz@tu-dresden.de
}

\begin{abstract}
Scanning acoustic microscopy (SAM) is an important tool in semiconductor industry for quality assurance. It is also used for non-destructive material characterization. Common acoustic microscopes only work with focused single element probes. The usage of phased array techniques in SAM can improve efficiency and enable new applications.

Annular Arrays allow the use of advanced measurement techniques like the simultaneous determination of thickness and sound velocity or the identification of curved interfaces. Furthermore, annular arrays enable the variation of the focus position, which allows the simultaneous examination of different depths. In this contribution we present our research in progress of the development of new multichannel microscopes and, in accordance with that, $40 \mathrm{MHz}$ annular probes based on 1-3 composites.
\end{abstract}

Key words: multi-channel scanning acoustic microscopy, material characterization, annular arrays, $40 \mathrm{MHz}$ 1-3-composite array

\section{Introduction}

SAM uses high frequency focusing ultrasound probes with frequencies between ten and a few hundred $\mathrm{MHz}$. The probe scans along a specific path, emits an acoustic wave at each probe position and receives the reflected signals (impulse-echo-mode).

Usual scanning modes are B-scans, C-scans and z-scans. A B-scan corresponds to a longitudinal cut of the investigated specimen. A CScan corresponds to a transverse section parallel to the scanning area. B- and C-Scans enable the detection of delaminations and incomplete bondings for quality assurance in electronical devices [1]. A z-scan enables the determination of the echo amplitude $V$ as a function of the distance between probe and specimen surface $z$, the so called $V(z)$-curve. The $V(z)$-curve is a fingerprint of the investigated specimen and allows material characterization [2].

For the localization of inclusions or for a C-scan of a specific depth via a time window, the sound velocity of the specimen has to be known. If the sound velocity is unknown, only qualitative conclusions about the location of determined structures can be done. For a structure with different layers, for instance an encapsulated die-stack component, the sound velocity of all layers has to be known for a correct reconstruction with an exact localization of inhomogeinities [3].

To achieve good resolution, the focus of the probe has to be located in the imaging depth by adjusting the z-position of the probe. Often, several depth levels of a specimen have to be investigated. For this, several time consuming scans are necessary.

Focusing with annular arrays may overcome this disadvantage. The time delayed excitation of the elements of an annular array enables the variation of the focus position without mechanical probe displacement and thus, the simultaneous investigation of several depths during a single scan.

Annular arrays also allow the application of advanced measurement techniques, like the simultaneous determination of thickness and sound velocity of layered structures [4] or the classification of reflecting interfaces in flat or curved [5]. The simultaneous determination of thickness and sound velocity provides a correct location of inhomogeinities and a new access to material characterization, in particular for hidden layers. The classification of reflecting interfaces can help to adjust the probe to achieve normal sound incident. 
Conventional SAMs only use single element probes. We present, therefore, a multichannel SAM to apply advanced measurement techniques based on annular arrays. Results of concluded and ongoing projects based on annular arrays are discussed and related to application possibilities in SAM. Also, high frequency, pre-focused phased array probe are not available commercially. By that reason, we develop a $40 \mathrm{MHz}$ 1-3 composite array.

\section{Advanced measurement techniques based on annular arrays}

Annular arrays allow the variation of the focus at a fixed probe position via time shifted excitation of the elements. This enables time saving by the simultaneous investigation of several layers with only a single scan process. Fig. 1 shows several C-scans of a power module [6]. The images of different depth levels of Fig. 1 b) - d) are achieved by a single scan with a $10 \mathrm{MHz}$ Annular Array. In Fig. $1 \mathrm{~d}$ ) the same level is mapped like in Fig. 1 a), which was recorded with a similar focusing probe. It can be seen, that the annular array achieves a comparable quality and resolution like the focusing probe.

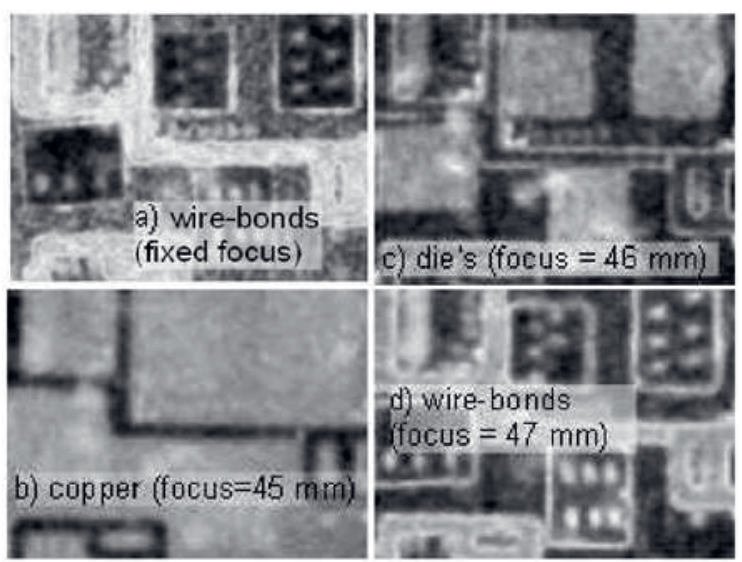

Fig. 1. C-scans of a power module in different levels [6]: a) c-scan of the wire-bonds with a focusing probe; $b-$ d) c-scans of different levels determined by focusing with an annular array in the corresponding depth

A further important application of SAM is material characterization. The above mentioned $V(z)$-curve technique requires a scan process. In contrast, annular arrays enable a nonscanning material characterization also of hidden layers. This is possible by an approach for the simultaneous determination of sound velocities and thicknesses, described in [4]. The approach is based on an iterative model, which uses synthetic focusing. To achieve synthetic focusing, each array element emits separately an ultrasonic wave and every array element receives the reflected waves. All recorded signals are time shifted by a set of calculated delay times and, afterwards, superposed. The delay times are calculated with a geometric model. In this model, the sound velocity of the considered layer is arbitrary chosen. The corresponding layer thickness results from time of flight between the interfaces in the considered layer and the chosen sound velocity.

The sound velocity in the considered layer is varied. The amplitude of the synthetic focused signal is highest, if the correct sound velocity is taken for the calculation of the delay times. Fig. 2 shows this procedure for the sound velocity determination of an epoxy layer behind a water delay line. The amplitude of the focused signal is shown as a function of the sound velocity. The actual sound velocity of the longitudinal wave can be read out from the maximum of this curve. The layer thickness results from the so determined sound velocity and the time of flight between the interfaces of the considered layer.

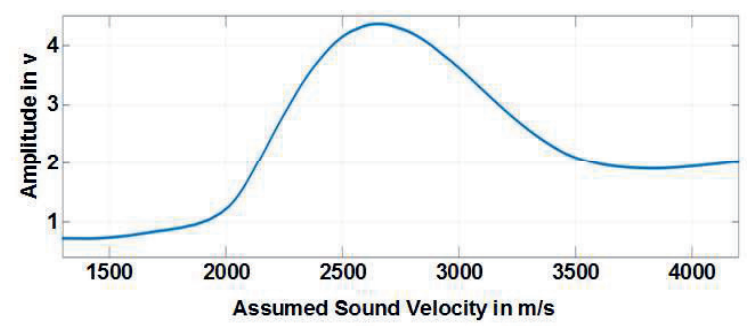

Fig. 2. Amplitude of the focused signal depending on the sound velocity taken to calculate the delay times

For material characterization, also the transversal wave velocity is required. This can be determined if longitudinal wave velocity and layer thickness are known. A condition for that is the generation of transversal waves. This can also be achieved with annular arrays due to mode conversion caused by inclined sound travel paths between the array elements. Fig. 3 a) shows a drawing of two sound travel paths: from emitting element 1 to the interface and back to the receiving element 1 (path 1) and from emitting element 2 to the interface and back to the receiving element 6 (path 2). In Fig. $3 \mathrm{~b}$ ) the corresponding signals in the region between the first and the second backwall reflection (BWR) can be seen. The signal from path 1 only shows the backwall echoes. In the signal of path 2, however, a shear wave reflection (SWR) appears between the both BWR. This shear wave reflection results by mode conversion on the backwall of the layer. Longitudinal wave velocity and layer thickness allow to calculate the sound travel paths. With these sound travel paths and the time of flight between the SWR and the first BWR, the transverse wave velocity can be determined. 
a)

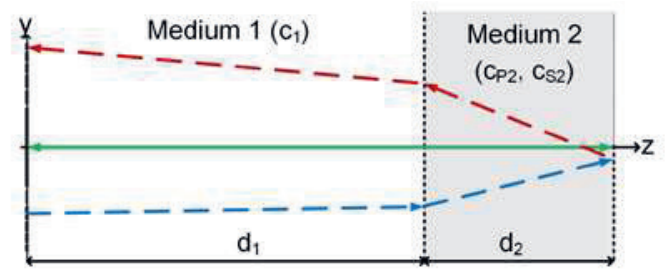

b)
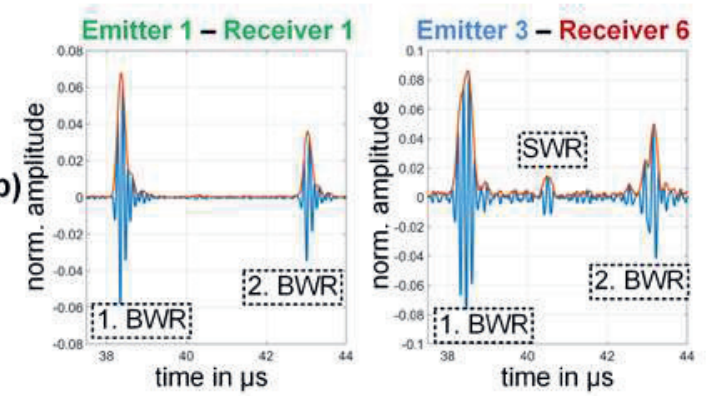

Fig. 3. a) Drawing oft he different sound travel paths between the emitting element to the interface and back to a receiving element; b) corresponding signals

Annular arrays also provide the possibility to identify curved surfaces. This is possible because the reflected sound field of an interface depends on its shape. The sound pressure distribution on the probe surface can be used to determine the shape of the interface by evaluating the directional pattern.

In [5], this effect was used to classify inclusions in disk and spherical shaped reflectors. For normal sound incident on a disk shaped reflector, the sound wave is reflected directional back to the probe (see Fig. 4 right). A spherical reflector, however, scatters the sound uniformly in all directions (see Fig. 4 left). This can be evaluated by measuring the amplitude differences between two annular elements. Large amplitude differences indicate flat reflectors, whereas small amplitude differences indicate spherical reflectors. This procedure can be used in a multi-channel SAM to detect curved interfaces. If a curved interface is identified, the probe alignment can be corrected with goniometers to assure normal sound incident and, therefore, to enable the investigation of structures behind curved interfaces.
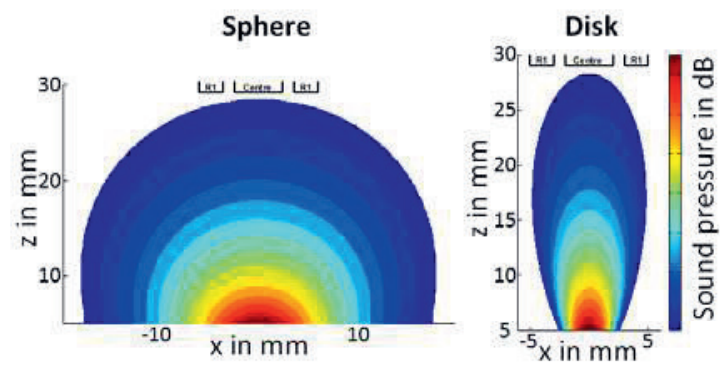

Fig. 4. Directional pattern of the reflected sound field from a sphere (left) and a disk (right)
All these examples demonstrate the possibilities of annular arrays. The challenge to apply these methods in SAM is the development of adequate sensors and electronics.

\section{Electronical devices}

Our scanning acoustic microscope is an inhouse construction [6]. It consists of a 16 true channel pulser/receiver unit, a 10 channel ADC (1GS/s, 8bit, Gage) and a set of linear and goniometer axis. The digitization is carried out without preprocessing, what enables the development of new evaluation algorithms.

The pulser/receiver unit has 16 independent true channels with variable gain and high precision dynamic pulse delay for receiving frequencies up to $100 \mathrm{MHz}$. Every channel can be excited seperately with time delayed pulses between $0-1.6 \mu$ s in 0.25 ns steps. The pulses have a width of 4-6 ns and the amplitude can be set between $20-350 \mathrm{~V}$. A 16:8 multiplexer allows to use all 16 channels with the 10 channel ADC.

A script-based interface for the measuring system enables quick changes of any setting such like trigger delay, record duration or pulse amplitude even while a scan is being performed. This enables to adjust the SAM to a wide range of measurement tasks. In addition to an automatically generated detailed protocol, the input-scripts allow immediate response on the recorded signals performing more detailed measurements and reducing the size of saved data.

With the help of high-precision linear axes, both, the probe and the test sample can be positioned quickly and accurately. Additional goniometers allow the variation of the probe orientation so that the inspection of curved structures is possible.

The actual prospective is the development of a 32 channel signal generator and receiver unit. The 32 channels enables to drive transducers with more elements for a more precise sound field forming. The planned integration of an arbitrary waveform generator improves the transducer's excitation using arbitrary shaped signals [7]. Arbitrary excitation signals can be used to generate, in addition to common pulses, quasi-harmonic signals (sine with finite number of wave-trains), coded signals or for an active damping of post-oscillations. Quasiharmonic signals enable the measurement of the phase-shifts between the reflected signals on the different probe elements. This allows, for example, the determination of the curvature radius of curved interfaces [8]. The active damping of post-oscillations is useful to 
evaluate reflections close to the probe. Furthermore, the active damping of postoscillations will lead to a higher bandwidth and therefore, a better resolution.

\section{Probes}

Phased array probes enable the electronic variation of the focus position. In contrast to matrix arrays, annular arrays reach this with few elements. Linear arrays have the drawback, that the focus is highly expanded in one direction, whereas with annular arrays a small circular focus can be realized.

Annular arrays with frequencies up to $60 \mathrm{MHz}$ can be found in literature ([9], [10]), however, with considerable drawbacks. In [9], design and fabrication of a $50 \mathrm{MHz}$ annular array with PZT as transducer material is discussed. The design of low frequency arrays with PZT is done by mechanical cutting. For high frequencies mechanical cutting is difficult, due to the small substrate thickness. By that reason, the element structure is realized by depositing the electrode pattern on the surface of the substrate. The disadvantage of this procedure is a significant mechanical cross-talk between the elements [11].

Design and Fabrication of a $40 \mathrm{MHz}$ Annular Array transducers based on PVDF is discussed in [10]. However, the achievable sound pressure with PVDF is low. The emitted acoustic energy is not high enough to investigate solid specimens.

Ultrasound arrays based on 1-3-composites have small mechanic cross talk and produce high acoustic energy. Unfortunately, 1-3composites based arrays with frequencies above $10 \mathrm{MHz}$ are not available commercially.

By this reason, our goal is to develop a prefocused $40 \mathrm{MHz}$ annular-array. The transducer material is fabricated by Fraunhofer-IKTS. In contrast to the traditional dice-and-fill procedure for the fabrication of 1-3 composites, a softmold-procedure, developed by [12], is used. Under consideration of certain width-heightratios, this process enables arbitrary sizes and shapes of the active pins and arbitrary spacing between the pins of the composite.

Sound field simulations are used to estimate the minimum number of elements and to optimize the space between the elements as well as the curvature for pre-focusing to focus in the aimed focusing range [13]. Fig. 5 (below) shows the sound field in water of a $35 \mathrm{MHz}-$ sparse annular array for focusing at $6 \mathrm{~mm}$. It has 10 active elements and a space between the elements of the same area as the active elements. The inner element has a diameter of $d=1.1 \mathrm{~mm}$. The element width decreases from the centre to the periphery. With a wavelength of $\lambda=43 \mu \mathrm{m}$ at $f=35 \mathrm{MHz}$, all elements are several multiples of the wavelength in width. Unlike linear arrays, annular arrays, therefore, have enormous advantages over linear arrays. The most important advantages are the higher element width and thereby, the reduction of the element number for the same aperture. In case of a sparse 10-element array, a focusing at 6 $\mathrm{mm}$ in water is possible without side lobes.

A conventional focused single element probe with $60 \mathrm{MHz}$ centre frequency (see Fig. 1 above) produce the same lateral $6 \mathrm{~dB}$ focus extension of $80 \mu \mathrm{m}$ compared to the $35 \mathrm{MHz}$ annular array. This is caused by the shorter focus distance and the larger aperture of the array.
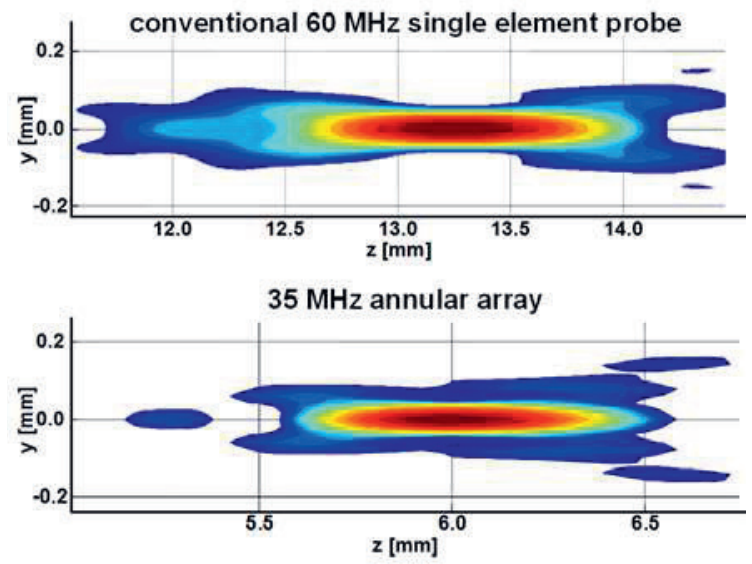

Fig. 5. Sound fields in water of a conventional, focusing microscopy probe ( $f=60 \mathrm{MHz} ; d=6.35 \mathrm{~mm}$; lens curvature: $r=10 \mathrm{~mm}$ ) and a flat annular array ( $f=35 \mathrm{MHz} ; 10$ elements; near field length of the single elements: $N_{\text {Single }}=7 \mathrm{~mm}$ )

In comparison to a plane sparse annular array, a curved array produces a shorter focal distance and thus, a higher lateral resolution. A minimal focus depth of $F O C=4 \mathrm{~mm}$ can be reached with a $35 \mathrm{MHz}$-curved sparse array with only 10 elements, an aperture of $5 \mathrm{~mm}$ and a curvature radius of $10 \mathrm{~mm}$. This results in a lateral focus extension $(6 \mathrm{~dB})$ of $60 \mu \mathrm{m}$.

For the manufacturing of the complete array transducer, the electrodes of the array are structured by sputter and lift-off technique. These electrodes shall be connected via bond wires to a printed circuit board, that acts as interconnector between the bond wires and the shielded cables to interface the control electronic. After the wire connection, the housing will be placed around printed circuit board and filled with epoxy resin that acts as backing and mechanical protection.

Two techniques are tested to apply the electrodes on the transducer material. At first, 
the lift off technique is tested. Hereby the mask consists of a photoresist, which is removed after sputtering the electrodes by using a solvent. The problem is to find an appropriate solvent, because common used solvents react with the 1-3 composite material. At second, a masking technique is applied, whereby a laser structured steel mask is used to produce the gap areas between the electrodes. The tests show, that the masking technique is qualified for the electrode application.

Actually, there remains a need for research for connecting the bond wires on the composite surface. Due to a too low hardness, the composite surface is locally deformed caused by pressure of the sonotrode. As the contact pressure is missing, no adhering connection between the bond wire and the electrode is processed.

\section{Summary and prospectives}

It has been shown, that annular arrays provide many advantageous features for SAM. A simultaneous inspection of several depth levels is possible. This leads to an improvement of the efficiency of SAM inspection and might be highly interesting for applications in semiconductor industry. To investigate one layer of a 12 inch wafer with good resolution, the measurement time is approximately 2 hours. For the investigation of the next generation of 18 inch wafers the measurement time would rise up to 5 hours.

For a quantitative evaluation the sound velocity of the investigated material has to be known. Annular arrays can be used to determine the sound velocities of longitudinal and transversal wave as well as the layer thicknesses of layered structures simultaneously. This enables, besides a better localizing of inclusions, material characterization, also for hidden layers.

This paper outlines our research in progress. A 16 channel pulser/receiver unit for frequencies up to $100 \mathrm{MHz}$ is in operation and was used to develop the mentioned measurement techniques. The actual goal is the new development of a multi-channel SAM with arbitrary signals for excitation. This will improve the possibilities for future research. The development of high frequency array probes based on 1-3 composites as transducer material is part of an ongoing project in cooperation with FraunhoferIKTS.

\section{References}

[1] S.Brand, S. Tismer, S. T. Moe, K. SchjølbergHenriksen, Non-destructive wafer-level bond defect identification by scanning acoustic microscopy, Microsystem Technologies 21:7,
1385-1394 (2015); doi: 10.1007/s00542-0142328-z

[2] Z. Yu, S. Boseck, Scanning acoustic microscopy and its applications to material characterization, Review of Modern Physics 67:4, 863-891 (1995); doi: 10.1103/RevModPhys.67.863

[3] N. Gust, Improvement of signal analysis for the ultrasonic microscopy, $P h D$ Thesis (2010)

[4] S. Kümmritz, M. Wolf, E. Kühnicke, Material Characterization of Layered Structures with Ultrasound, Physics Procedia 70, 330-333 (2015); doi: 10.1016/j.phpro.2015.08.218

[5] S. Kümmritz, M. Wolf, E. Kühnicke, Investigation of Embedded Structures in Media with Unknown Acoustic Properties, QNDE Proceedings (2016)

[6] N. Gust, F. Schellhorn, High frequency 16 channel ultrasonic microscope for annular arrays, IEEE International Ultrasonics Symposium Proceedings (2012); doi: 10.1109/ULTSYM.2012.0609

[7] A. Juhrig, S. Kümmritz, M. Wolf, E. Kühnicke, A multichannel ultrasound pulser with arbitrary signal excitation for scanning acoustic microscopy, VIII ${ }^{\text {th }}$ International Workshop NDT in Progress 12 (2015)

[8] M. Lenz, J. Sorber, E. Kühnicke, G. Gerlach, T. Rödig, H. G. Trier, Non-scanning measurement of local curvature with an ultrasound annular array, IEEE International Ultrasonics Symposium Proceedings (2009); doi: 10.1109/ULTSYM.2009.0676

[9] J. A. Brown, C. E. M. Démoré, G. R. Lockwood, Design and Fabrication of Annular Arrays for High-Frequency Ultrasound, IEEE Transactions on Ultrasonics, Ferroelectrics, and Frequency Control 50:8, 1010-1017 (2004); doi: 10.1109/TUFFC.2004.1324405

[10] J. A. Ketterling, O. Aristizábal, D. H. Turnball, F. L. Lizzi, Design and Fabrication of a $40-\mathrm{MHz}$ Annular Array Transducer, IEEE Transactions on Ultrasonics, Ferroelectrics, and Frequency Control 52:4, 672-681 (2005); doi: 10.1109/TUFFC.2005.1428050

[11] M. Lenz, N. Gust, M. Wolf, E. Kühnicke, T. Rödig, Transducer Characterization by Sound Field Measurements, IEEE Transactions on Ultrasonics, Ferroelectrics, and Frequency Control 60:5, 998-1009 (2013); doi: 10.1109/TUFFC.2013.2658

[12] A. Gebhardt, A. Schönecker, R. Steinhausen, T. Hauke, W. Seifert, H. Beige, Fine scale 1-3 composites fabricated by the soft mold process: Preparation and modeling, Ferroelectrics 241:1, 67-73 (2000); doi: 10.1080/00150190008224976

[13] E. Kühnicke, Design of curved annular arrays for high-frequency imaging, AIP Conference Proceedings 1096, 816 (2009); doi: $10.1063 / 1.3114340$ 